\title{
AREA-PRESERVING HOMEOMORPHISMS OF THE OPEN DISK WITHOUT FIXED POINTS
}

\author{
STEVE ALPERN
}

(Communicated by R. Daniel Mauldin)

\begin{abstract}
D. G. Bourgin has proved that every measure-preserving orientation-preserving homeomorphism of the open two-dimensional disk $D$ has a fixed point. He suggested that the "result is perhaps valid even if the condition of orientability preservation be dropped." We show that on the contrary there exist fixed point free homeomorphisms of $D$ which preserve any given finite nonatomic locally positive Borel measure. Examples are also constructed in all higher dimensions.
\end{abstract}

According to Brouwer's Fixed Point Theorem every continuous map of the closed $n$-ball into itself must have a fixed point, although of course there need be no interior fixed point even for homeomorphisms. In particular it is easy to construct homeomorphisms of the open $n$-ball which have no fixed points. However D. G. Bourgin [Bo] has used Brouwer's result on translation fields [Br] to prove the following: Every measure-preserving orientation-preserving homeomorphism of the open twodimensional ball has a fixed point. The preserved measure may be Lebesgue measure or any finite nonatomic Borel measure which is positive on open sets. In that paper Bourgin suggested that his theorem might be extended to orientation reversing homeomorphisms of the open two-ball and perhaps also to higher dimensions. The latter conjecture has been settled in the negative by Asimov [As], who found fixed point free orientation preserving diffeomorphisms of the open $n$-disk, $n \geq 3$, which preserve Lebesgue measure. Similar questions for symplectic diffeomorphisms have been considered by Colvin and Morrison $[\mathbf{C M}, \mathbf{C}]$.

However the existence of (orientation reversing) measure-preserving homeomorphisms of the open two-ball without fixed points is apparently still open. In this paper we settle this question of Bourgin by proving moreover that there is an orientation-reversing homeomorphism of the closed two-dimensional ball which preseves Lebesgue measure and has no interior fixed point. This result applies equally well to other measures, higher dimensions and, for $n \geq 3$, to orientation-preserving homeomorphisms. Our main tool is the "Homeomorphic Measures Theorem" of Oxtoby and Ulam [OU]. For smooth examples, the similar result of Moser [Mo] could be used instead.

Instead of balls, we will find it easier to work on the $n$-cubes $I_{n}=\left\{x\right.$ in $R^{n} ;-1 \leq$ $\left.x_{i} \leq+1\right\}$. Denote by $S^{n-1}$ and $D_{n}$ the boundary and interior of $I_{n}$, respectively. Let $I_{n}^{+}$and $I_{n}^{-}$be the upper and lower half-cubes of $I_{n}$ determined by $x_{n} \geq 0$ and $x_{n} \leq 0$, and let their common face $x_{n}=0$ be denoted by $F_{n}$. We will identify

Received by the editors March 23, 1987.

1980 Mathematics Subject (Classification (1985 Revision). Primary 54H25; Secondary 57N05, $58 \mathrm{C} 30$. 
$I_{n-1}$ with $F_{n}$ by the embedding $p=p_{n}: I_{n-1} \rightarrow F_{n}$ given by $p\left(x_{1}, \ldots, x_{n-1}\right)=$ $\left(x_{1}, \ldots, x_{n-1}, 0\right)$. Every homeomorphism $g$ of $I_{n-1}$ determines a homeomorphism $\phi(g)$ of $F_{n}$ by $\phi(g)=p g p^{-1}$. Let $e$ denote the identity map and $\alpha$ be the reflection of $R^{n}$ about the hyperplane $x_{n}=0, \alpha\left(x_{1}, \ldots, x_{n-1}, x_{n}\right)=\left(x_{1}, \ldots, x_{n-1},-x_{n}\right)$. Let $M(B)$ be the set of all nonatomic finite Borel measures on $B$ which are positive on open sets and zero on the boundary of the ball $B$. In particular, let $m$ denote Lebesgue measure of the appropriate dimension. Let $H\left(I_{n}\right)$ be the group of all selfhomeomorphisms of $I_{n}$ and let $H^{f}\left(I_{n}\right)$ be the subset of homeomorphisms whose restriction to the boundary $S^{n-1}$ is equal to $f$. For $\mu$ in $M\left(I_{n}\right)$, let $H_{\mu}\left(I_{n}\right)$ and $H_{\mu}^{f}\left(I_{n}\right)$ be the subsets of $\mu$-preserving homeomorphisms. We can now state

HOMEOMORPhiC MEASURES THEOREM [OU]. Let $B$ be an $n$-ball and let $f$ be any homeomorphism of $B$ onto itself. Then given any two measures $\mu_{1}$ and $\mu_{2}$ in $M(B)$, with $\mu_{1}(B)=\mu_{2}(B)$, there is a homeomorphism $h: B \rightarrow B$ which equals $f$ on the boundary of $B$ and satisfies $\mu_{2}(h(A))=\mu_{1}(A)$ for all Borel subsets $A$ of $B$.

LEMMA 1. Given any $g$ in $H^{e}\left(I_{n-1}\right)$ there is an $f$ in $H\left(I_{n}^{+}\right)$such that $\phi(g)$ is the restriction of $f$ to $F_{n}$ and $f$ is the identity on all the other faces of $I_{n}^{+}$.

PROOF. Every homeomorphism of a closed ball which fixes the boundary is isotopic to the identity. Hence there is an isotopy $G: I_{n-1} \times[0,1] \rightarrow I_{n-1}$ from $g$ to the identity $e$. That is, $G$ is continuous and each $g_{t}(x)=G(x, t)$ is a homeomorphism, with $g_{0}(x)=g(x)$ and $g_{1}(x)=x$. The lemma is established by defining $f\left(x, x_{n}\right)=\left(G\left(x, x_{n}\right), x_{n}\right)$, where $x=\left(x_{1}, \ldots, x_{n-1}\right)$.

LEMMA 2. Given any homeomorphism $g$ in $H^{e}\left(I_{n-1}\right)$ there exists a homeomorphism $r$ in $H_{m}^{\alpha}\left(I_{n}\right)$ such that $r\left(I_{n}^{+}\right)=I_{n}^{-}, r\left(I_{n}^{-}\right)=I_{n}^{+}$, and $r$ equals $\phi(g)$ on $F_{n}$.

ProOF. Let $f$ be the "extension" of $g$ to $I_{n}^{+}$given by Lemma 1. Apply the Homeomorphic Measures Theorem to $f$ and $I_{n}^{+}=B$, with $\mu_{1}=\mu_{2}=m$, to obtain an $m$-preserving homeomorphism $h$ of $I_{n}^{+}$which is the identity on all the faces of $I_{n}$, except for $F_{n}$, where it equals $\phi(g)$. Define $r$ by $r(x)=\alpha h(x)$ if $x_{n} \geq 0$ and $r(x)=h(\alpha(x))$ if $x_{n} \leq 0$.

LEMMA 3. If there is a homeomorphism $g$ in $H^{e}\left(I_{n-1}\right)$ which has no interior fixed point then there is an orientation-reversing homeomorphism $r$ in $H_{m}^{\alpha}\left(I_{n}\right)$ which has no interior fixed point.

PROOF. Given $g$, let $r$ be the homeomorphism obtained using Lemma 2. If $x$ is an interior fixed point of $r$, then $x$ must be in $F_{n}$, and $p^{-1}(x)$ is a fixed point of $g$. Since this is contrary to assumption, $r$ has no interior fixed point.

THEOREM 1. For $n \geq 2$ there exists an orientation-reversing volume-preserving homeomorphisms $r$ in $H_{m}^{\alpha}\left(I_{n}\right)$ which has no interior fixed points.

PROOF. According to Lemma 3, we need only establish the existence of homeomorphisms of $I_{n}, n=1,2, \ldots$, which fix the boundary and have no interior fixed points. This is of course trivial. (Take for example the homeomorphism $g\left(x_{1}, \ldots, x_{n-1}, x_{n}\right)=\left(x_{1}, \ldots, x_{n-1},\left(\left(x_{n}+1\right)^{2} / 2\right)-1\right)$.) 
COROLLARY 1. For any $n \geq 2$ and any measure $\mu$ in $M\left(I_{n}\right)$, there exists an orientation-reversing $\mu$-preserving homeomorphism $s$ of $I_{n}$ which has no interior fixed point.

ProOF. Let $r$ be the homeomorphism given by Theorem 1. Apply the Homeomorphic Measures Theorem to the case $B=I_{n}, \mu_{1}=\mu, \mu_{2}=m$, and $f=e$. Let $h$ in $H^{e}\left(I_{n}\right)$ be the resulting homeomorphism with $m(h(A))=\mu(A)$ for all Borel subsets $A$. Then the homeomorphism $s=h^{-1} r h$ is as required.

COROLlARY 2. Let $\mu$ be any finite nonatomic measure on the open $n$-ball $D$, $n \geq 2$, which is positive on all nonempty open sets. Then there is an orientationreversing, $\mu$-preserving homeomorphism $t: D \rightarrow D$ which has no fixed point.

Proof. Let $t$ be the restriction of the homeomorphisms of Corollary 1 to $D$.

COROLLARY 3. For $n \geq 3$ there is an orientation-preserving volume-preserving homeomorphism of $I_{n}$ which has no interior fixed point.

PROOF. Fix any $n \geq 2$ and let $r$ be the $m$-preserving orientation reversing homeomorphism of $I_{n}$ given by Theorem 1 , which has no interior fixed point. Define $g$ in $H\left(I_{n+1}\right)$ by $g\left(x, x_{n+1}\right)=\left(r(x),-x_{n+1}\right)$, where $x$ denotes $\left(x_{1}, \ldots, x_{n}\right)$. It is clear that $g$ is orientation-preserving and $m$-preserving. If a point $\left(x, x_{n+1}\right)$ were an interior fixed point of $g$, then we would have $x_{n+1}=0$ and $x$ would be an interior fixed point of $r$, contrary to assumption.

The obstruction to extending Corollary 3 to dimension two is the lack of any orientation-reversing homeomorphism of the one-dimensional cube $[-1,+1]$ which has no interior fixed point. Of course Corollary 3 can be extended to arbitrary measures in $M\left(I_{n}\right)$ or to the open cube $D_{n}$ using the same constructions as in Corollaries 1 and 2.

(Added in proof, September 1987) D. Mauldin has informed me of two additional references regarding the fixed point theorem I credited to Bourgin. In [Mn, pp. 952-953] D. Montgomery briefly observes that this fixed point result is a consequence of Brouwer's translation theorem, and Andrea [An, Proposition 1.5] writes out a proof of this result, which he credits to Montgomery.

\section{REFERENCES}

[An] S. Andrea, On homeomorphisms of the plane which have no fixed points, Abh. Math. Sem. Univ. Hamburg 30 (1967), 61-74.

[As] D. Asimov, On volume-preserving homeomorphisms of the open $n$-disk, Houston J. Math. 2 (1976), 1-3.

[Bo] D. G. Bourgin, Homeomorphisms of the open disk, Studia Math. 31 (1968), 433-438.

[Br] L. E. J. Brouwer, Beweis des ebenen Translationssatzes, Math. Ann. 72 (1912), 37-54.

[CM] M. Colvin and K. Morrison, A symplectic fixed point theorem on open manifolds, Proc. Amer. Math. Soc. 84 (1982), 601-604.

[Mn] D. Montgomery, Measure preserving transformations at fixed points, Bull. Amer. Math. Soc. 51 (1945), 949-953.

[M] K. Morrison, Symplectic flows on the open ball, J. Differential Equations 46 (1982), 59-62.

[Mo] J. Moser, On the volume elements of a manifold, Trans. Amer. Math. Soc. 120 (1965), 286294.

[OU] J. Oxtoby and S. Ulam, Measure preserving homeomorphisms and metrical transitivity, Ann. of Math. (2) 42 (1941), 874-920.

Department of Mathematics, London School of ECONOMics, London WC2A 2AE, UNITED KINGDOM 